\title{
Yet Another Access Point
}

\author{
Richard Gass \\ Intel Research Pittsburgh \\ richard.gass@intel.com
}

\author{
Christophe Diot \\ Thomson \\ christophe.diot@thomson.net
}

This article is an editorial note submitted to CCR. It has NOT been peer reviewed.

The authors take full responsibility for this article's technical content. Comments can be posted through CCR Online.

\begin{abstract}
Increasing popularity in personal access points and travel routers has begun to rise with the advent of smaller and more battery conscious devices. This article introduces the YAAP (Yet Another Access Point), an open source software that enables ad-hoc infrastructure services in the absence of network connectivity. The YAAP provides a familiar way to connect users to each other and provides a set of useful services. We list and describe its applications, explain how it can be used, provide details about the code, and point readers to where it can be downloaded.
\end{abstract}

Categories and Subject Descriptors: D.2.2 [Design Tools and Techniques]: User Interfaces

General Terms: Design, Experimentation

Keywords: Personal Proxy

\section{INTRODUCTION}

Accessing services from the Internet has become commonplace since the introduction of smartphones and hand-held devices. Users can enjoy near seamless connectivity to online resources practically anywhere via cellular networks. Unfortunately, wireless data plans are expensive, may require a dedicated monthly service plan, and data rates are slow. Community $802.11 \mathrm{Wi}-\mathrm{Fi}$ networks are potential sources of bandwidth. Despite the fact that $802.11 \mathrm{Wi}-\mathrm{Fi}$ access points are ubiquitous in most urban areas, they are not utilized to their full potential by passers-by.

Mobile users depend a great deal on infrastructure and the services they provide. In areas of no connectivity, i.e., areas with no Wi-Fi or cellular coverage, mobile users are cut-off from the world and are left in solitude, not able to send email or exchange data in an easy manner, even if the intended destination is in the same room. Ad-hoc networks are one way of creating a local network for users to share, but due to different incompatibilities between operating systems, it is not yet trivial to setup and could potentially pose security problems. Today, the universally accepted way to transfer files due to a lack of infrastructure services is by way of the USB storage key.

Three years ago we designed and implemented the YAAP (Yet Another Access Point) [6] to overcome these issues and to allow experimentation without having to modify end user client systems while researching in-motion networking. The YAAP is a small form factor computer (about the size of a cigarette box) that offers local storage, multiple network interfaces, and offers locally connected users a familiar and easy way to allow their devices to communicate with one another in a private and secure fashion.

With gaining popularity to similar devices such as MiFi [4] that uplink over cellular networks, travel routers available from most popular network infrastructure vendors [5], and the potential interest of the community to reuse code to support new use cases such as in-motion networking, home networks, or ad-hoc opportunistic communication, we decided to make the community aware of the existence of the freely available YAAP source code.

\section{WHAT IS THE YAAP}

The YAAP was prototyped on the Stargate platform ${ }^{1}$ which is based on a $400 \mathrm{MHz}$ Xscale processor. This platform was selected because the smartphones capable of supporting the YAAP were not yet available or prohibitably expensive. However, the YAAP software is hardware independent and would run on any smartphone or mobile device that supported the minimal set of services required by the software (described in section 3).

The YAAP software includes three main applications that are accessed through a regular web browser:

1) Chat: A web based chat server that did not require external connectivity or any client side modifications. A user can post to a message board viewable by all YAAP users as well as send instant messages to individual users or to a group.

2) File exchange: An easy and familiar web based way to upload files that are stored on the local storage of the YAAP. Users uploading files can configure permissions to allow their individual files to be viewable by only a specific user, a group of users, or available for public consumption.

3) Proxy services: Users are able to enter queries into a local search proxy to view previously searched items that are related. If no related items are found, the search request is queued and fulfilled as soon as the YAAP finds external connectivity.

The YAAP can be used in a combination of any of the four primary configurations:

- Router mode: Allows a connection to the Internet via an Ethernet cable and allows multiple clients to share that link over an 802.11 interface.

- Access point mode: The YAAP behaves as a wireless infrastructure access point allowing multiple clients to connect and share information via direct communication or through a simple web interface.

\footnotetext{
${ }^{1}$ http://www.xbow.com
} 
- Wireless Client mode: Similar to router mode but the uplink is through a second wireless interface.

- Proxy mode: This mode acts as a transparent proxy and allows asynchronous communication between the mobile client and the Internet through short opportunistic contacts with access points encountered while transiting from one place to another.

All of these modes are configurable through a administrative web interface. The admin interface also allows the owner of the YAAP or administrator to add or delete users, reset passwords, and configure network interfaces.

A typical usage scenario could be a group visiting another company's site. If guest wireless is unavailable due to security issues with the hosting company, the group could turn on the YAAP and easily begin sharing information with each other, documenting events at the meeting, and privately communicating with one another. Another situation could be a business traveller in a hotel. The traveler pays for wired access in her room and realizes that only her laptop can be connected to the Internet. The traveler instead plugs the YAAP into the wired connection and begins to share the connection with all of her devices (e.g. laptop, phone, and PDA)

The YAAP operates simply by flipping a switch to turn it on. After a short time to boot, it configures itself to one of the user selected modes. The owner can then associate and connect just as with any other infrastructure access point. Once connected to the YAAP, she can simply open a web browser to be automatically redirected to the main login page.

Newly associated users to the YAAP arrive at the main login page and are instructed to register by creating a username and password. If guest access is allowed by the YAAP owner and registration is successful, the user is able log into the YAAP site. User configurable profile options, like invisibility, can be selected in order hide the presence of the user from other users on the system.

The proxy mode of the YAAP allows a user, not currently connected to the Internet, to make web search requests via the interface. If no similar results from previous searches are found, the search is placed in a queue on the YAAP to be served later. While the user is transiting from one place to another, the YAAP (in your pocket or your bag) constantly searches for community wireless access points the users is subscribed to (e.g., the FON network[1]). Once the YAAP successfully connects to an access point, it immediately begins to process the queued web requests, forwarding them to the user configurable search engine of choice. The top three search results are stored (search depth is also configurable) and can be accessed when the user reconnects to the YAAP.

Another feature of the proxy is that if you have a YAAP that is configured to act as an Internet proxy, it will bundle data to allow bulk transfers to the client. This allows the online YAAP to send data in bulks to the frequently disconnected client enabling more data to be transferred at each encounter, reducing delays incurred by chatty protocols like HTTP[3].

\section{DOWNLOAD THE YAAP}

The following services are required to the get the YAAP up and running:
- Web server: A web server with PHP is required to serve up the YAAP web pages. We run the Apache web server.

- DHCP server: A DHCP server is needed to assign IP addresses to clients connecting to the YAAP. We used udhcpd, which is a small, fully functional, DHCP server

- NAT: All client connections are NAT'ed, so the YAAP needs to be able forward packets to clients on the internal network. We have installed iptables and use IP masquerading to handle this.

- Captive portal: This is needed to intercept all incoming requests and redirect users to the main YAAP login page. We wrote a small DNS responder to intercept all incoming DNS requests and redirect clients to the IP address of the YAAP.

The source code for the YAAP is hosted at SourceForge[2]. This bulk of the code is intended to be dropped into the web root of a Stargate and be served by a web server. The latest release provides all the web functionality needed to begin sharing files, chatting, and asynchronously downloading web content.

Proxy code that is used to perform bulk transfers between the client and online YAAPs will be added to the repository as soon as it has been properly field tested.

\section{SUMMARY}

The YAAP was designed to easily provide drop-in, adhoc infrastructure services. It offers an easy and familiar way for users to share files and chat, with or without infrastructure support. The YAAP is also able to take advantage of opportunistic contacts encountered while moving between locations. The code can be used on stand-alone handheld devices, smartphones, and Internet proxies allowing experimentation without modifications to the client. We have made the source code publicly available on Sourceforge[2] to allow the community to support and develop for these new use cases such as in-motion networking or ad-hoc opportunistic communication.

\section{REFERENCES}

[1] Community wireless by FON. http://www.fon.com.

[2] YAAP software download. http://sourceforge.net/projects/yaap, December 2005.

[3] Gass, R., Scott, J., And Diot, C. Measurements of in-motion 802.11 networking. In WMCSA '06: (HotMobile) (Semiahmoo Resort, Washington, USA, 2006), pp. 69-74.

[4] Novatel Wireless InC. http://investor.novatelwireless.com/ releasedetail. cfm?ReleaseID=353263, December 2008.

[5] Roseberry, C. Top 7 travel wireless routers. http://mobileoffice.about.com/od/ laptopaccessories/tp/pocketrouters.htm.

[6] YAAP, Yet Another Access Point. Poster, Intel Braunschweig Grand Opening, http://pittsburgh.intel-research.net/ rgass/ posters/yaap/YAAP-Braunschweig2006.pdf. 